High-Pressure Tailored Compression: Controlled Thermodynamic Paths

Jeffrey H Nguyen, Daniel Orlikowski, Frederick H. Streitz, John A. Moriarty, Neil C. Holmes

October 24, 2005

Journal of Applied Physics 
This document was prepared as an account of work sponsored by an agency of the United States Government. Neither the United States Government nor the University of California nor any of their employees, makes any warranty, express or implied, or assumes any legal liability or responsibility for the accuracy, completeness, or usefulness of any information, apparatus, product, or process disclosed, or represents that its use would not infringe privately owned rights. Reference herein to any specific commercial product, process, or service by trade name, trademark, manufacturer, or otherwise, does not necessarily constitute or imply its endorsement, recommendation, or favoring by the United States Government or the University of California. The views and opinions of authors expressed herein do not necessarily state or reflect those of the United States Government or the University of California, and shall not be used for advertising or product endorsement purposes. 


\title{
High-Pressure Tailored Compression: Controlled Thermodynamic Paths
}

\author{
Jeffrey H. Nguyen, Daniel Orlikowski, Frederick H. Streitz, John A. Moriarty, Neil C. Holmes \\ Lawrence Livermore National Laboratory \\ Livermore, California 94551, USA
}

(Dated: October 21, 2005)

\begin{abstract}
We have recently carried out novel and exploratory dynamic experiments where the sample follows a prescribed thermodynamic path. In typical dynamic compression experiments, the samples are thermodynamically limited to the principal Hugoniot or quasi-isentrope. With recent developments in the functionally graded material impactor, we can prescribe and shape the applied pressure profile with similarly-shaped, non-monotonic impedance profile in the impactor. Previously inaccessible thermodynamic states beyond the quasi-isentropes and Hugoniot can now be reached in dynamic experiments with these impactors. In the light gas-gun experiments on copper reported here, we recorded the particle velocities of the $\mathrm{Cu}-\mathrm{LiF}$ interfaces and employed hydrodynamic simulations to relate them to the thermodynamic phase diagram. Peak pressures for these experiments were on the order of megabars, and the time-scales ranged from nanoseconds to several microseconds. The strain rates of the quasi-isentropic experiments are approximately $10^{4} \mathrm{~s}^{-1}$ to $10^{6} \mathrm{~s}^{-1}$ in samples with thicknesses up to $5 \mathrm{~mm}$. Though developed at a light-gas gun facility, such shaped pressure-profiles are also feasible in principle with laser ablation or magnetic driven compression techniques allowing for new directions to be taken in high pressure physics.

PACS numbers: 62.20.-x; 62.50.+p; 64.70.-p
\end{abstract}


The ability to attain extreme pressure and temperature conditions has given investigators in fields as diverse as biology, condensed matter physics, and earth and planetary sciences the tools to explore material behavior at megabar pressures and at thousands of degrees [1-13]. With these tools, they have simulated the physical conditions in the Earth's core and planetary interiors $[1,2]$, probed a wide range of high pressure and temperature material properties [5-10], synthesized novel materials [11, 12], and solved long standing physics problems such as the metallization of hydrogen [13]. These extreme conditions were achieved through three main techniques: static, shock and quasi-isentropic compressions [13-24]. Since the pioneering work by P. W. Bridgman [25], advances in diamond anvil cell technology have pushed the peak pressure by static compression from 200 kbars to more than 4 megabars [21, 22]. Similarly, shock compression and quasi-isentropic compression techniques [13-20] can load samples to megabar pressures in a fraction of a nanosecond to microseconds.

Future advances will likely push the peak pressure higher and extend the pressure loading time. However, these techniques will continue to be limited to a portion of the high pressure phase diagram by their characteristic loading rate and a single thermodynamic path. In particular, static compression yields continuous states on an isotherm (or an isochore, when heating), with a slow loading rate of $\dot{\epsilon}<10^{1} \mathrm{~s}^{-1}$. Shock compression rapidly loads a sample at strain rates of $\dot{\epsilon} \gtrsim 10^{9} \mathrm{~s}^{-1}$ to a single state on the Hugoniot - a locus of shock compression states. Current quasi-isentropic compression techniques constrain samples to lie near an isentrope, with strains rates of around $\dot{\epsilon} \approx 10^{5} \mathrm{~s}^{-1}$ to $\dot{\epsilon} \approx 10^{8} \mathrm{~s}^{-1}$ [17-20]. With these vastly different strain rates varying as much as 10 orders of magnitude, there is no a priori reason to assume that the high pressure and temperature data gathered with these differing techniques can be directly compared [1]. What is needed is a less constrained approach that can produce highly compressed states, as well as afford continuity between the thermodynamic paths and time scales.

We describe herein a series of dynamic compression experiments designed and performed with an approach that not only removes the constraint of a specific thermodynamic path, but also helps to bridge the gap in loading rates between a static and shock compression experiment. This method allows for flexibility in designing the applied pressure profile through various sequential combinations of shock, quasi-isentropic compression, controlled release and constant pressure. The resulting pressure profiles in the samples have time-scales 
ranging from nanoseconds to microseconds.

The ability to dynamically apply an arbitrary shaped pressure pulse to a sample has been realized through an impactor (Fig. 1) that has a corresponding arbitrary shock impedance profile along the thickness of the impactor (impedance $=c \rho_{\circ}$, where $\mathrm{c}$ is the sound speed and $\rho_{\circ}$ the density). An increasing (decreasing) impedance profile in the impactor imparts an increasing (decreasing) applied pressure to the sample system. Abrupt increases (decreases) in impedance can be used to generate shock (rarefaction) waves that propagate into the sample. To achieve these types of sequential impedances in the impactor, fine layers $(\lesssim$ $200 \pm 20 \mu \mathrm{m}$ thick) were assembled into a functionally graded material (FGM) impactor. The individual layers are themselves composed of $\mathrm{Al}$ (or $\mathrm{Mg}$ ) and $\mathrm{W}$ powders $(\leq 5 \mu \mathrm{m}$ in particle size), mixed in the appropriate proportions to produce the desired impedance. Sintered powders yielded the highest densities $(2.7 \mathrm{~g} / \mathrm{cc}$ to $\lesssim 15 \mathrm{~g} / \mathrm{cc}$ for Al-W powders; $1.7 \mathrm{~g} / \mathrm{cc}$ to $15 \mathrm{~g} / \mathrm{cc}$ for $\mathrm{Mg}-\mathrm{W}$ powders). Lower density impedance layers were created by embedding the powders in a resin matrix (final densities of $1.2 \mathrm{~g} / \mathrm{cc}$ to $8 \mathrm{~g} / \mathrm{cc}$ ) or a foam matrix (densities of $0.01 \mathrm{~g} / \mathrm{cc}$ to $2.7 \mathrm{~g} / \mathrm{cc}$ ). In practice, we restricted the lowest densities to about $0.1 \mathrm{~g} / \mathrm{cc}$ so that the integrity of the impactor can be maintained during launch (where acceleration can be as high as $80,000 \mathrm{~ms}^{-2}$ ). By employing any or all of these techniques, impactors with varying densities ranging from $0.1 \mathrm{~g} / \mathrm{cc}$ to $15 \mathrm{~g} / \mathrm{cc}$ over a thickness of several millimeters were produced.

A series of $\mathrm{Cu}$ experiments were carried out on a two-stage light-gas gun with the FGM impactors, and simulations were performed to understand the resulting thermodynamic paths. The impactors were launched at velocities between 1 and $4.5 \mathrm{~km} / \mathrm{s}$ towards a $\mathrm{Cu}$ target (typically a $\mathrm{Cu}$ disk a few-mm thick and flat to better than $5 \mu \mathrm{m}$, tamped with a $10 \mathrm{~mm}$ thick single crystal of LiF) (Fig. 1). We have successfully launched low-density FGM impactors at velocities as high as $6 \mathrm{~km} / \mathrm{s}$. A velocity interferometer (VISAR) [26] was used to record the particle velocity, $U_{p}$, at the $\mathrm{Cu}-\mathrm{LiF}$ interface (Fig. 1).

In order to draw a connection between the experimental $U_{p}$ and the system's thermodynamic path through pressure, volume, and temperature (PVT) space, one- and twodimensional hydrodynamic simulations were performed for the complete experimental setup [27]. A typical set-up consists of a FGM impactor striking a $\mathrm{Cu}$ target sample with a tamped $\mathrm{LiF}$ window. The necessary equations-of-state (EOS) for each layer within the FGM were developed through iterative simulations of a series of experiments with impactors 
of various impedance profiles and launch velocities. In addition, some individual layers or sections of layers were launched at $\mathrm{Cu}$ targets to develop the corresponding EOS set for the impactor. The simulations presented here included adjustments made to the densities in the simulated FGM impactor to account for manufacturing variations. This was performed to study the sensitivity of the thermodynamic path to density perturbations. EOS information for $\mathrm{Cu}$ and $\mathrm{LiF}$ were taken from the standard material LLNL EOS library. Finally, the strength of the target and window was modeled through the Steinberg-Guinan model [28].

In Fig. 2, we present two representative quasi-isentropic experiments on $\mathrm{Cu}$ obtained from two similar FGM impactors with monotonic impedance profile launched at two different velocities. The particle velocities, $U_{p}$, at the $\mathrm{Cu}-\mathrm{LiF}$ interface are seen to rise smoothly, with no evidence of shock, for a period of 2 to $3 \mu$ s before reaching peak velocities of 0.073 and $0.05 \mathrm{~cm} / \mu \mathrm{s}$. The linear increase in the observed $U_{p}$ represents a nearly constant strain rate during the experiment, albeit perturbations from this linear increase do occur (e.g., the variation at $\mathrm{t}=2 \mu \mathrm{s}$ of V3787 in Fig. 2). These perturbations have little effect due to their small relative amplitude. We estimate the strain rate to average $3-5 \times 10^{4} \mathrm{~s}^{-1}$ for these shots by using hydrodynamic simulation results. These strain rates are similar to or are an order of magnitude lower than other quasi-isentropic compression techniques [17-20]. At the presented $\dot{\epsilon}$ in metals, the sample is expected to be near local thermal equilibrium, since the thermal relaxation times for particle interactions are significantly faster: $10^{-13} \mathrm{~s}$ (electron-phonon), $10^{-12} \mathrm{~s}$ (phonon-phonon), and $10^{-12} \mathrm{~s}$ (electron-electron) [29]. Specifically in Fig. 2(inset), we show the related temperature-pressure (T-P) path transversed in the $\mathrm{Cu}$ sample during the experiment, as given by hydrodynamic simulation. The calculated path agrees to better than $0.2 \%$ with the isentrope, while the calculated temperature of the sample at peak pressure of $70 \mathrm{kbar}$ is already $1 \%$ lower than that on the Hugoniot. In pressuredensity space, the simulations agree even better to the isentrope (not shown). In experiments that approach the Mbar regime, simulations indicate temperatures significantly lower than those on the Hugoniot, but slightly above the isentrope, due to the effect of dissipation mechanism (strength) in the sample.

The ability to control the time scale of the experiment extends to the quasi-isentropic pressure-release as well. By creating a FGM impactor with a constant initial impedance followed by a slowly decreasing impedance, we can control the rate of pressure release. As demonstrated in Fig. 3, the rate of pressure release due to the controlled rarefaction wave 
is a factor of three slower than if the rarefaction had been induced by a single-impedance impactor in a typical shock experiment. Such a controlled pressure release could be used to mimic a Taylor wave in one-dimension.

By employing FGM impactors with monotonic impedance profiles, we have demonstrated in these experiments (Figs. 2-3) the capability to monotonically move through the thermodynamic phase space, i.e., quasi-isentropes from an ambient initial state or an initial state on a Hugoniot. However, we can exploit the flexibility in FGM impactor manufacturing to expand the attainable phase space within a single experiment. This is achieved by designing a FGM impactor with a corresponding non-monotonic impedance profile to drive the sample with a non-monotonic pressure-time history. In general, a sequential set of compressions, rarefactions, shocks, or constant pressures can be produced in the sample resulting in a set of discrete and/or continous thermodynamic states. The first experiment of this character is presented in Fig. 4. For this experiment, the impactor was comprised of a uniform, high-impedance layer followed by a low-impedance and monotonically increasing impedance layers. The effective thermodynamic path transversed by the $\mathrm{Cu}$ involves a shock, a pressure-release and a quasi-isentropic compression. The obtained states - shocked, released, and finally compressed - are sequentially demarcated in relation to the $\mathrm{U}_{\mathrm{p}}$ record (points B, C, and D, respectively, in Fig. 4) and also in the T-P plane taken from calculation (inset Fig. 4).

In the experiment discussed above (Fig. 4), the quasi-isentrope was the same for the release as for the compression in a single experiment. However, a family of quasi-isentropes are obtainable. In Fig. 5, we present an experiment demonstrating such capability. The FGM was designed in similar manner to the previous experiment (Fig. 4) except for the introduction of a step of increased impedance in the impactor prior to recompression. This design produced a second shock jumping the system to a new state, from which the system is recompressed (Fig. 5, top panel). The $\mathrm{Cu}$ target passes through two thermodynamically parallel quasi-isentropes, broadening the accessible T-P plane through an increase in temperature within a pressure range.

In some experiments, it is desirable to maintain periods of constant pressure at particular states. This can be achieved by increasing the impedance layer thickness in the impactor, as demonstrated in Fig. 5(bottom panel). Again similar to Fig. 4, particular $U_{p}$ are held for approximately $0.15 \mu \mathrm{s}$ during the release (points B and $\mathrm{C}$ ). The calculated path of the $\mathrm{Cu}$ 
system in T-P plane is similar to Fig. 4(inset), however with time spent at particular states along the quasi-isentropes.

Such thermodynamic paths, in addition to a choice in strain rate, can be chosen in advance by the experimentalist and designed into the FGM impactor. This customization of impedance profile allows access to a broad region of phase space. Indeed, the high T-P quasi-isentrope (BCD in Fig. 4) obtained here could not have been attained from ambient conditions using any current conventional techniques [14-20, 23, 30].

These results help us to understand the connection between the observed particle velocities and the thermodynamic paths and states. This FGM impactor, or similar compression profiles at other facilities, can be used to extend the strain-rate of quasi-isentropic compression experiments, and broaden the thermodynamic phase space available. Hence, in the future this will allow a bridging of the time-scale gap between static and dynamic compressions. In addition, with appropriate design through hydrodynamic simulations, one can explore complex and previously unattainable sequences of thermodynamic paths controlled by combinations of shock, quasi-isentropic compression, release, and static pressure in one dynamic experiment. With this technique, one can therefore dynamically probe those timedependent features of a phase diagram, such as phase transitions[30], or investigate the dynamics of material response along various thermodynamic paths (strength hardening). Currently, this FGM impactor is being applied to a study of liquid-solid phase transitions that investigates strain-rate effects, kinetics and possible hysteresis effects, as well as timescales [31]. These are just a few cases in which new directions may be stimulated for high pressure physics. Impactor manufacturing process is continually being improved and experiments are being carried out to address issues such as planarity of the impedance layers, and impedance matching of the impactor sections. These improvements and alternative techniques of analysis such as backward integration and Lagrangian analysis will be reported in subsequent publications.

We would like to acknowledge useful discussions with R. Chau and R. Minich and the dedicated efforts of W. Hall, N. Hinsey, M. O'Shell, E. Loughnane, J. van Lue, J. van Lewen, K. Lewis, L. Raper, K. Stickle, S. Weaver, and E. White. This work was performed under the auspices of the U.S. Department of Energy by University of California Lawrence Livermore National Laboratory under contract No. W-7405-Eng-48. 
[1] J. H. Nguyen and N. C. Holmes, Nature 427, 339 (2004).

[2] W. B. Hubbard et al., Phys. of Plasmas 4, 2011 (1997).

[3] H. Herberhold et al., J. Mol. Biol. 330, 1153 (2003).

[4] A. Sharma et al., Science 297, 5580 (2002).

[5] H. Cynn, J. E. Klepeis, C. S. Yoo and D. A. Young, Phys. Rev. Lett. 88, 135701 (2002).

[6] V. V. Struzhkin et al., Science 2981213 (2002).

[7] M. I. Eremets, V. V. Struzhkin, H. K. Mao, and R. J. Hemley, Science 293272 (2001).

[8] V. V. Struzhkin, R. J. Hemley, H. K. Mao, and Y. A. Timofeev, Nature 390382 (1997).

[9] S. C. Schmidt, D. S. Moore, D. Schiferl, and J. W. Shaner, Phys. Rev. Lett. 50, 661 (1983).

[10] N. C. Holmes, W. J. Nellis, W. B. Graham and G. E. Walrafen, Phys. Rev. Lett. 55, 2433 (1985).

[11] F. P. Bundy et al., Carbon 34, 141 (1996).

[12] V. Iota, C. S. Yoo, and H. Cynn, Science 283, 1510 (1999).

[13] W. J. Nellis, S. T. Weir, and A. C. Mitchell, Phys. Rev. B 59, 3434 (1999).

[14] L. M. Barker, High-pressure Quasi-isentropic Impact Experiments. Shock Waves Cond. Matt. J. R. Asay, R. A. Graham and G. K. Straub, (eds.) (Elsevier Sci. Pub., Amsterdam)(1984).

[15] Chhabildas, L. C., Asay, J. R. \& Barker, L. M. SAND88-0306 Sandia Nat. Lab., Albuquerque, New Mexico(April 1988).

[16] Q. Shen et al., Acta Phys. Sinica, 51, 1759 (2002).

[17] C. A. Hall, Phys. Plasma 7, 2069 (2000).

[18] C. A. Hall et al., Rev. Sci. Instru. 72, 3587 (2001).

[19] D. B. Reisman et al., J. Appl. Phys. 89, 1625 (2001).

[20] J. Edwards et al., Phys. Rev. Lett. 92, 075002 (2004).

[21] M. Eremets, High Pressure Experimental Methods, Oxford University Press, Oxford, 1996.

[22] A. L. Ruoff, H. Xia, and Y. Vohra, Rev. Sci. Instrum. 61, 3830-3 (1990).

[23] Y. B. Zel'dovich and Y. P. Raizer, Physics of Shock Waves and High-Temperature Hydrodynamic Phenomena. (Academic Press, New York, 1967).

[24] M. Knudson et al., J. Appl. Phys. 94, 4420 (2003).

[25] P. W. Bridgman, Proc. Am, Acad. Arts Sci. 81 167-251, (1952). 
[26] L. M. Barker and R. E. Hollenbach, J. Appl. Phys. 43, 4669 (1972).

[27] CALE (C-language-based Arbitrary Lagrangian Eulerian) is a two-dimensional hydrodynamic code developed at LLNL.

[28] D. J. Steinberg, S. G. Cochran, and M. W. Guinan, J. Appl. Phys. 51, 1498 (1980); D. J. Steinberg and C. M. Lund, J. Appl. Phys. 65, 1528 (1989).

[29] D. C. Wallace, Phys. Rev. B 22, 1477 (1980).

[30] G. E. Duvall and R. A. Graham, Rev. Mod. Phys. 49, 523 (1977).

[31] J. H. Nguyen, D. Orlikowski, F. H. Streitz, N. C. Holmes and J. A. Moriarty, Specifically prescribed dynamic thermodynamic paths and resolidification experiments, Shock Comp. Cond. Matt. M. D. Furnish, L. C. Chhabildas, and R. S. Hixson, (eds.) (AIP Conf. Proc., Melville, New York)(2004) pp. 1225-1230. 

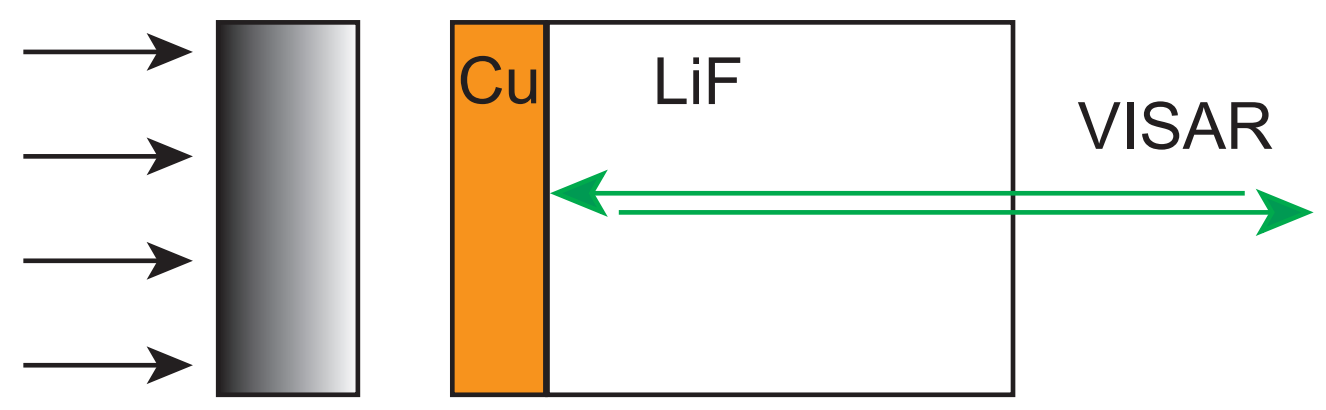

FIG. 1: Illustration of a typical experimental set-up. The FGM impactor is launched toward a stationary $\mathrm{Cu}-\mathrm{LiF}$ target at velocities up to $4.5 \mathrm{~km} / \mathrm{s}$. A VISAR [26] is used to record particle velocity $\left(\mathrm{U}_{p}\right)$ at the $\mathrm{Cu}-\mathrm{LiF}$ interface. 


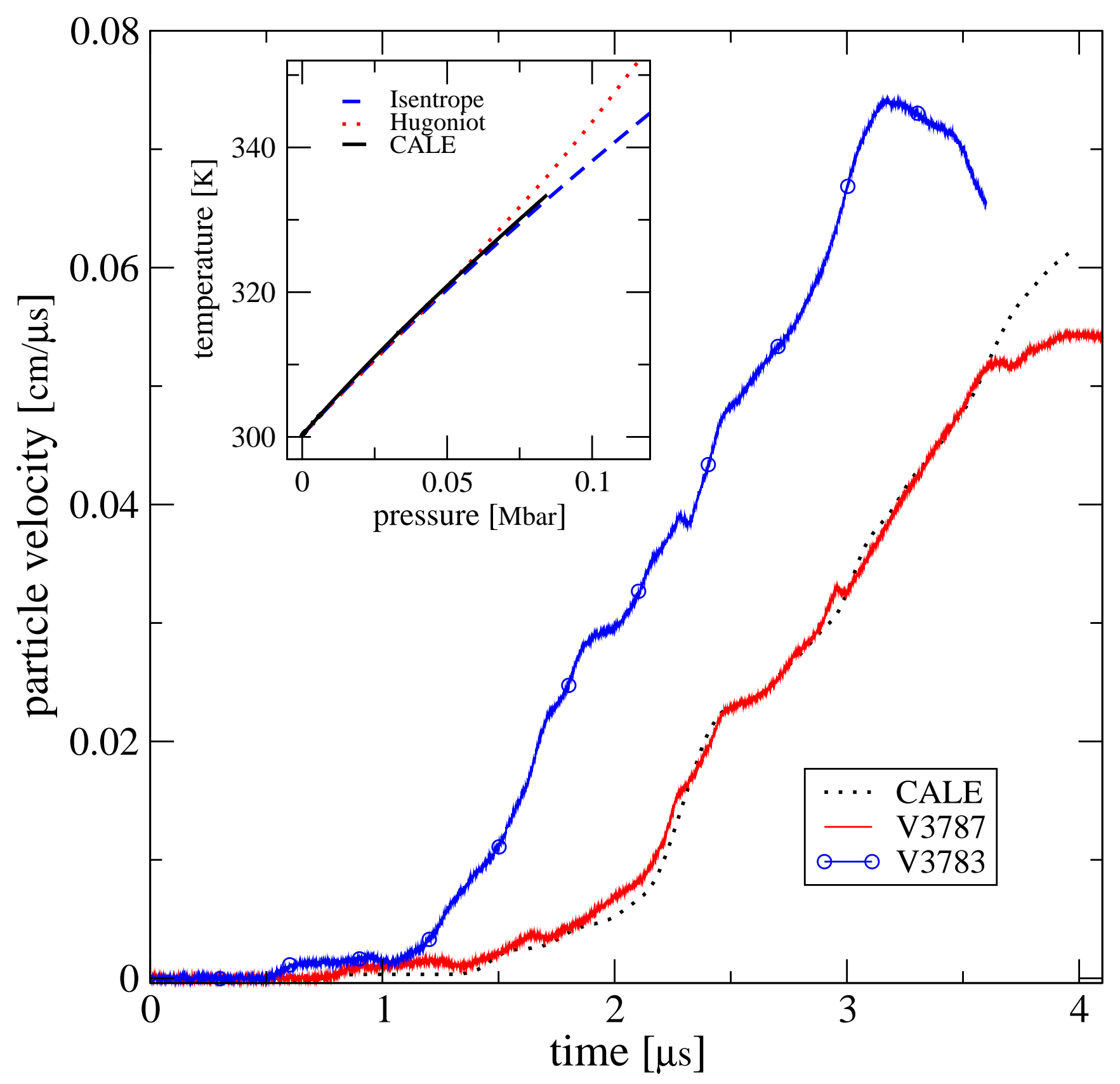

FIG. 2: Typical particle velocity $\left(\mathrm{U}_{p}\right)$ record from a compression experiment at the light-gas gun [impactor velocity $1.0 \mathrm{~km} / \mathrm{s}$ (V3787) (solid line) and $1.2 \mathrm{~km} / \mathrm{s}$ (V3783)] is shown (line-circle). A hydrodynamic calculation (CALE) [27] models the impactor composition and simulates the experiment in one-dimension (dotted line). The deviation of the experiment from calculation at $0.05 \mathrm{~cm} / \mu$ s is due to rarefraction (release) waves from the side of the $\mathrm{Cu}$-target. Inset: a comparison is made between the thermodynamic path from the modeled experiment, Hugoniot, and the quasiisentrope for $\mathrm{Cu}$. The calculation is within $0.2 \%$ (numerical error) of the quasi-isentrope and is approximately $1 \%$ of the Hugoniot at the highest obtained pressure. The strain is $6 \%$. 


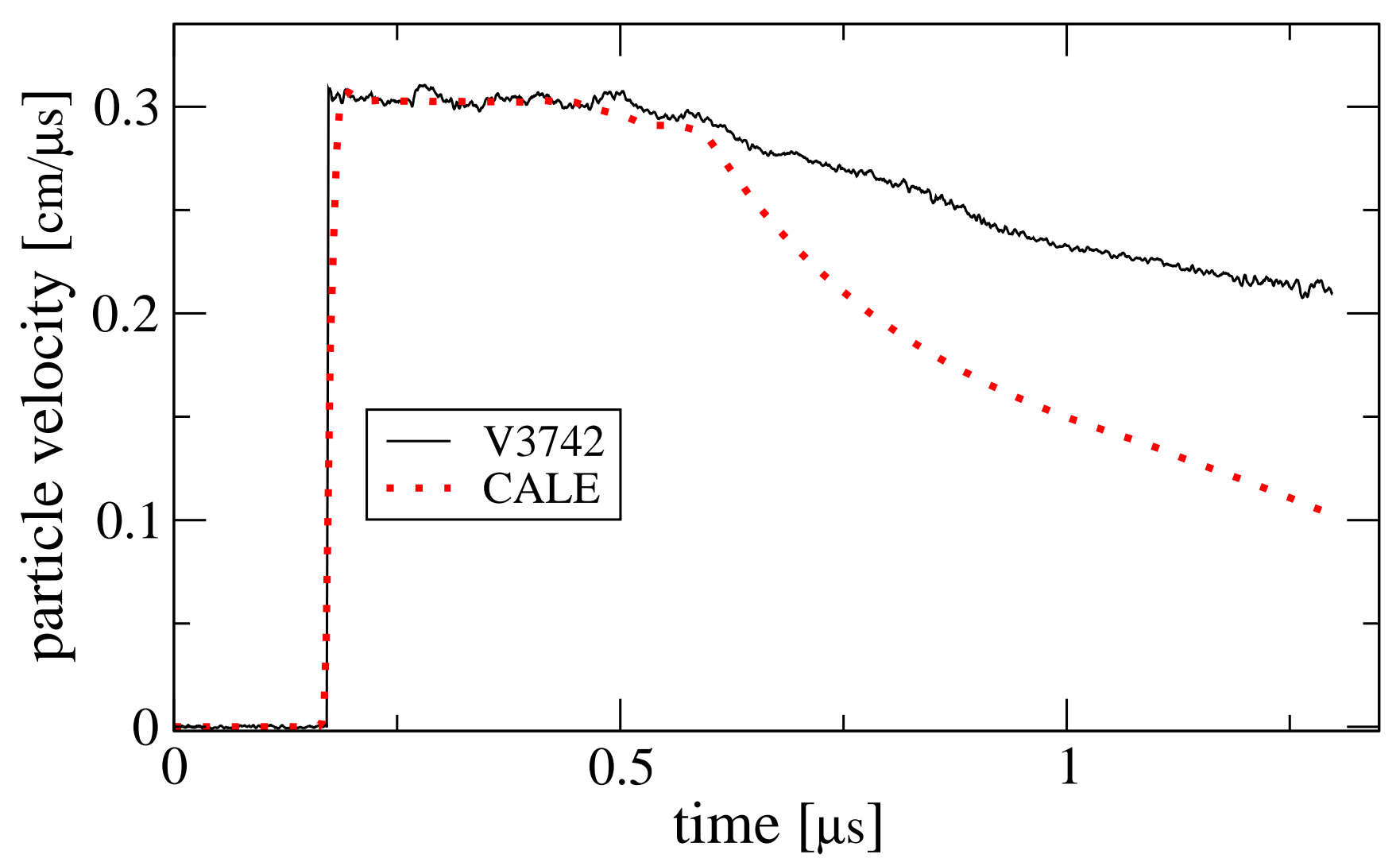

FIG. 3: The experimental $\mathrm{U}_{p}$ record $(\mathrm{Cu} / \mathrm{LiF}$ interface, solid-line) is shown for a controlled rarefraction (release) wave at a different strain-rate as compared with a simulated rarefraction wave (dotted-line) that would normally originates from the free-surface of the non-impacting end of a single-density impactor in a standard shock experiments. 


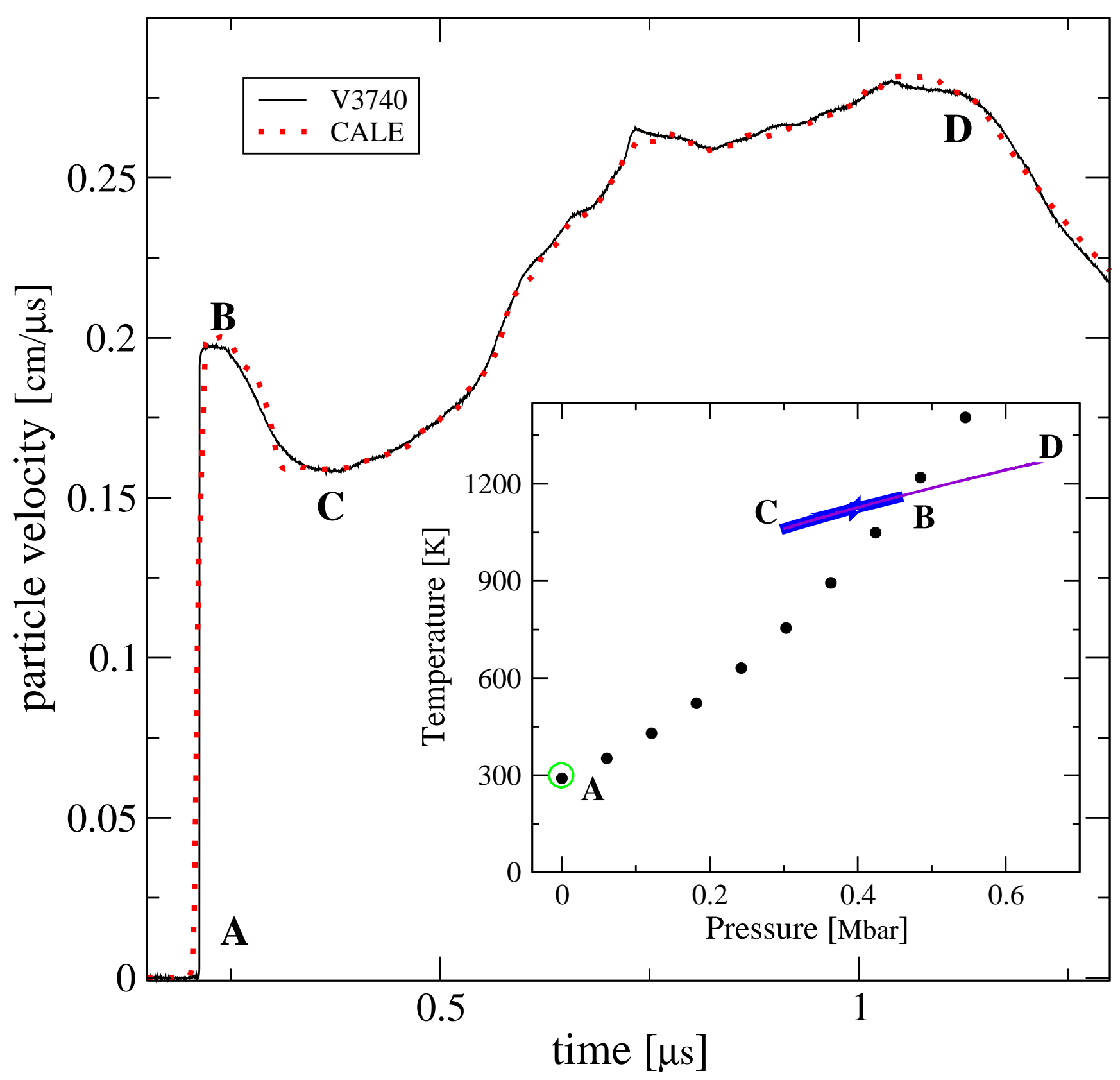

FIG. 4: $\mathrm{A} \mathrm{U}_{p}$ record $(\mathrm{Cu} / \mathrm{LiF}$ interface) shows a $\mathrm{Cu}$ system that is shock-compressed $(\mathrm{A}-\mathrm{B})$, released (B-C), quasi-isentropically compressed (C-D) and finally released. The hydrodynamic simulation (dotted-line) overlays the experimental (solid line) $\mathrm{U}_{p}$. (Inset) From simulation, the corresponding path in the (T-P) plane. The dotted line denotes the principal Hugoniot. 

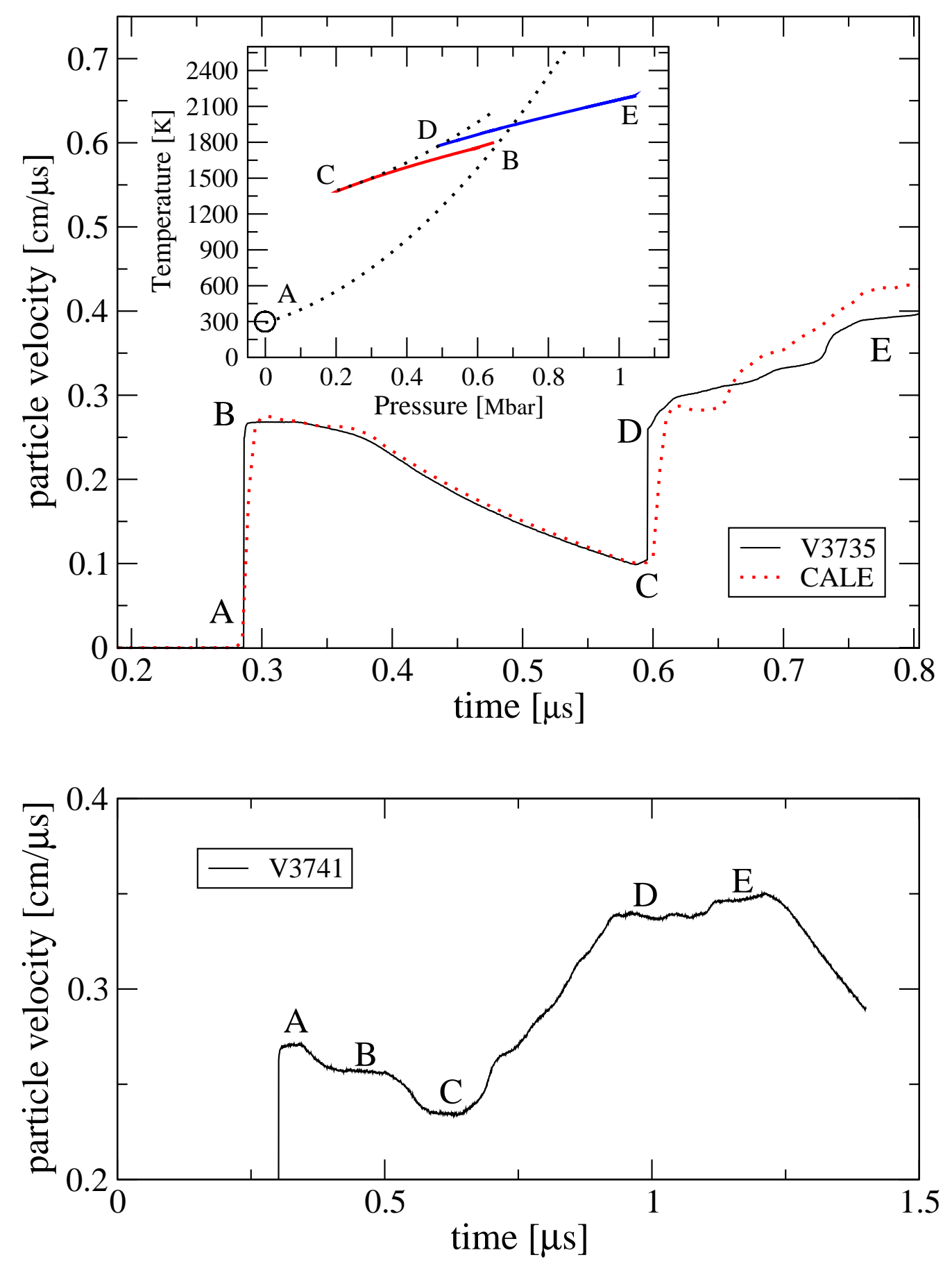

FIG. 5: (Top panel) An experiment demonstrating multiple quasi-isentropic compression paths is shown. Solid line (dotted line) denotes experimental measurements (hydro-simulation) of particle velocity $\mathrm{U}_{p}$. (Inset) the corresponding T-P path from the simulation with the associated Hugoniots (dotted lines). The system is shock-compressed (A-B), released (B-C), re-shocked (C-D), and quasi-isentropically compressed (D-E). (Bottom panel) A particle velocity trace produced through a sequential impedance arrangement in the impactor shows that the $\mathrm{Cu}$ system was controlled to and held at each of the labeled states via a shock to point (A), controlled releases (A-B and B-C), and quasi-compression (C-D). 